\title{
Angiomatoid Fibrous Histiocytoma in the Forearm: A Case Report
}

\author{
Hatim Mohammed A Alshareef ${ }^{1}$, Ahmed Hassan A Alhasan ${ }^{2}$, Mohammed G. Felemban ${ }^{1}$, Ashwaq Mohammed J \\ Almalki $^{3} \&$ Amer Mohammed A Alshareef ${ }^{4}$ \\ ${ }^{1}$ Orthopedic Department, King Fahad armed forces hospital, Jeddah, KSA \\ ${ }^{2}$ College of Medicine, University of Jeddah, Jeddah, KSA \\ ${ }^{3}$ College of Medicine, Taif University, Taif, KSA \\ ${ }^{4}$ Ibn Sina National College, Jeddah, KSA \\ Main Author: Hatim Mohammed Alshareef, E-mail: dr.hatim.alshareef@gmail.com \\ Correspondence: Ahmed H.Alhasan, College of Medicine, University of Jeddah, Jeddah, KSA. E-mail: \\ Ahmed_med14x@outlook.sa
}

Received: August 29, 2016

Accepted: September 29, 2016 Online Published: November 6, 2016

doi:10.5539/cco.v6n1p36

URL: http://dx.doi.org/10.5539/cco.v6n1p36

\begin{abstract}
Background: Angiomatoid fibrous histiocytoma $[\mathrm{AFH}]$ is a rare soft tissue tumor most commonly occurring in children, adolescents, and young adults. It is considered to be a tumor of intermediate malignancy because of its less aggressive course. The extremities are the most common site of involvement, followed by the trunk and head and neck.

Case presentation: A 28-Year-old male presented with a right forearm mass that has been present for 2 years. There was no associated fever, no trauma, no night pain and no constitutional symptoms. On examination he had a forearm mass dorsally measuring $2 \times 3 \mathrm{~cm}$, firm, rounded, mobile with no skin changes and full range motion joint above and joint below. Lab and imaging studies done which showed soft tissue mass, multilobulated complex heterogeneous with increased vascularity. Patient underwent biopsy and surgical excision.

Conclusion: Angiomatoid fibrous histiocytoma is a neoplasm of intermediate biologic potential. Most often, the extremities are the most common site of involvement, followed by the trunk and head and neck of children and young adults. Its rarity may lead to misdiagnosis as either a reactive lesion or a benign or higher-grade tumor. This tumor has a relatively rare metastasis and its overall clinical outcome is excellent. It is clinically important to accurately diagnose this tumor so that wide local excision may be performed and patients may be appropriately monitored and followed up.
\end{abstract}

Keywords: Angiomatoid fibrous histiocytoma, Soft tissue, Tumor

\section{Introduction}

Angiomatoid fibrous histiocytoma (AFH) is an uncommon, low-grade malignant soft tissue tumor of uncertain histogenesis. It most commonly occurs in the deep dermis or subcutis of the extremities in children and young adults (Enzinger, 1979; Costa \& Weiss, 1990; Fanburg-Smith, 2002).

AFH was originally described as angiomatoid "malignant" fibrous histiocytoma in 1979 by Enzinger, but has since been renamed due to its very infrequent malignant behaviour (Athanasou, 1999). WHO in 2002 removed $\mathrm{AFH}$ as a subtype of malignant sarcoma and placed it in the category of tumors of uncertain differentiation (Fletcher, 2006).

This lesion is rare and has the potential for local recurrence and metastasis, so it is imperative to consider this lesion in the differential diagnosis of a soft tissue mass in a child or adolescent (Bauer et al., 2012).

In this study, we report on a case of angiomatoid fibrous histiocytoma located deep in subcutaneous tissue of the right forearm in a 26-year-old gentleman.

\section{Case Report}

A 28 Year old male was referred by vascular surgery with a right forearm mass that has been present for 2 years. Initially presented to vascular surgery with history of gradual swelling forearm mass. He had background history 
of pulmonary Tuberculosis 6 years ago that has resolved since. There was no associated fever, no trauma, no night pain and no constitutional symptoms. He was then referred to the orthopedic department for further evaluation. On examination he had a forearm mass dorsally measuring $2 \times 3 \mathrm{~cm}$, firm, rounded, mobile with no skin changes and full range motion joint above and joint below.

\section{Lab investigations showed:}

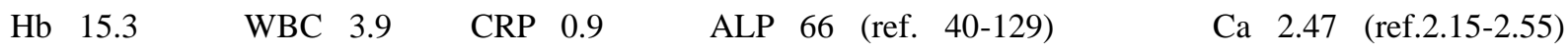

Phosphate 1.18 (ref. $0.80-1.45$ )

\section{Imaging Studies: X-rays:}
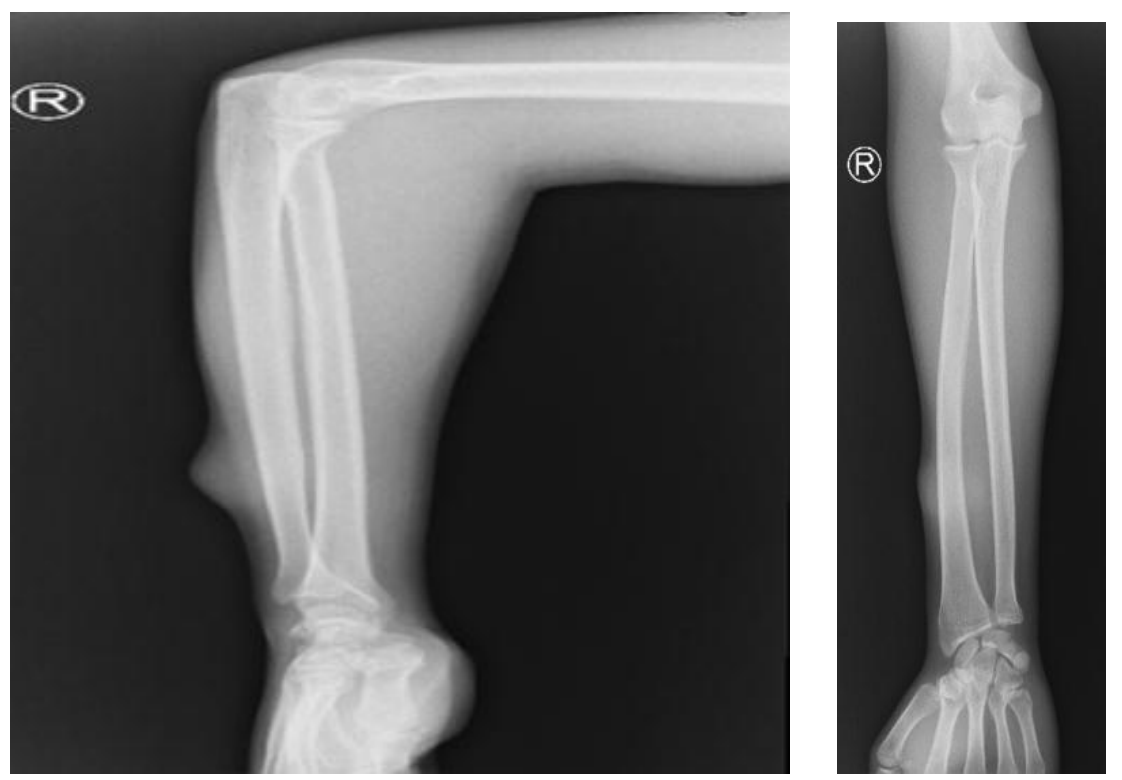

Figure $1 \&$ 2. X-Ray Forearm Showing Soft Tissue Mass with No Periosteal Reaction

Imaging /US:

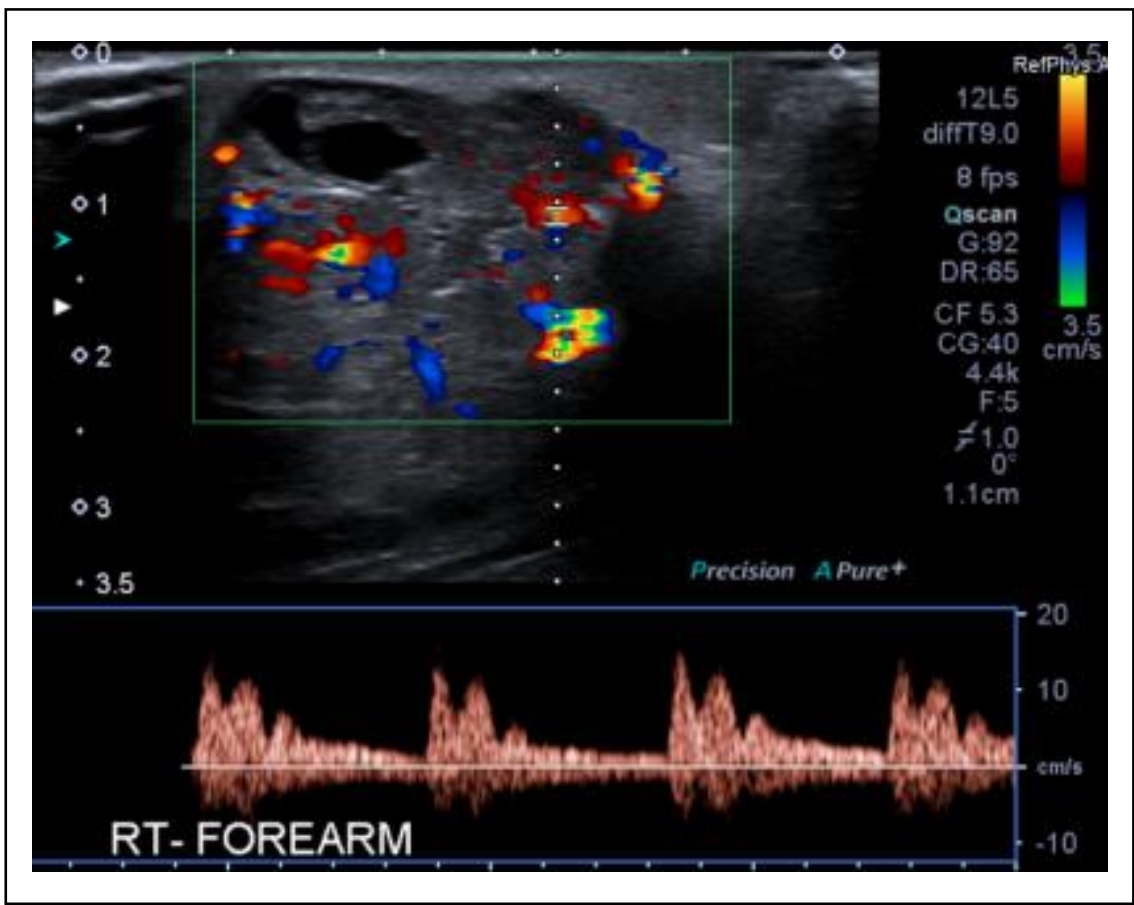

Figure 3. Ultrasound Forearm: Multilobulated Complex Heterogeneous Mass with Increased Vascularity 
Imaging/ MRI:
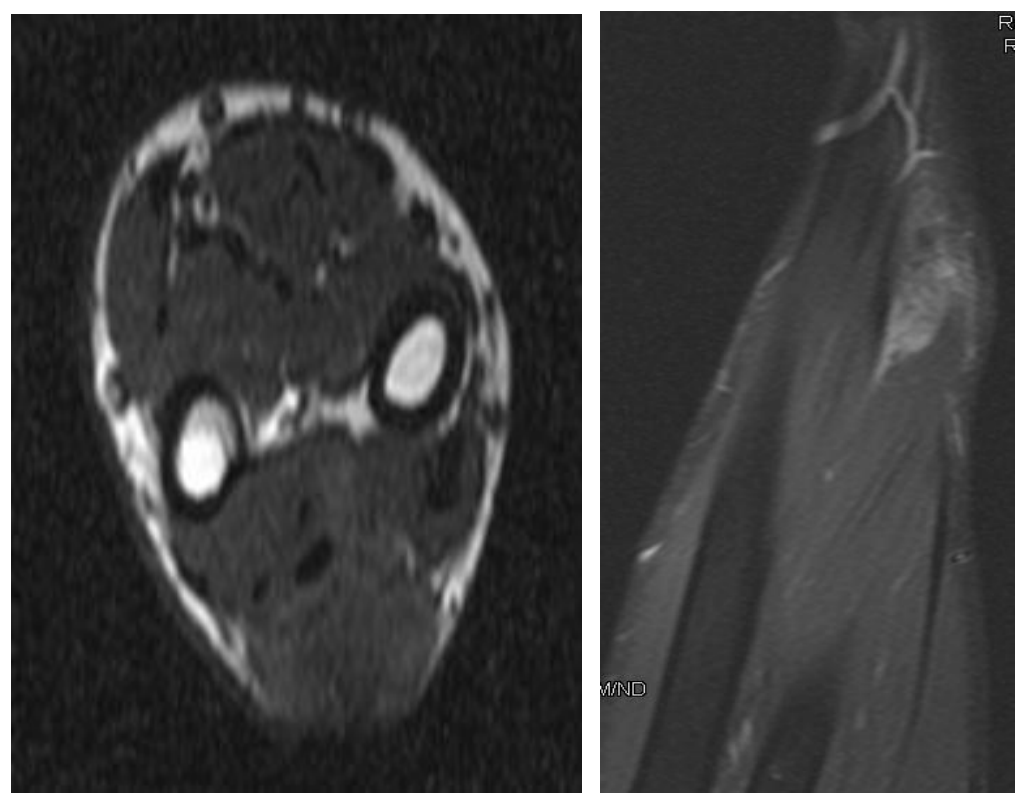

Figure 4\&5. MRI right forearm: Distal forearm soft tissue mass lesion, differential diagnosis can include vascular lesion like haemangioma or vascular malformation, versus solid lesions including myofibroma, atypical lipoma, nodular fasciitis

Further investigations were ordered. CT angiography was also suggestive of a vascular mass such as AVM, hemangioma vs neoplasm.
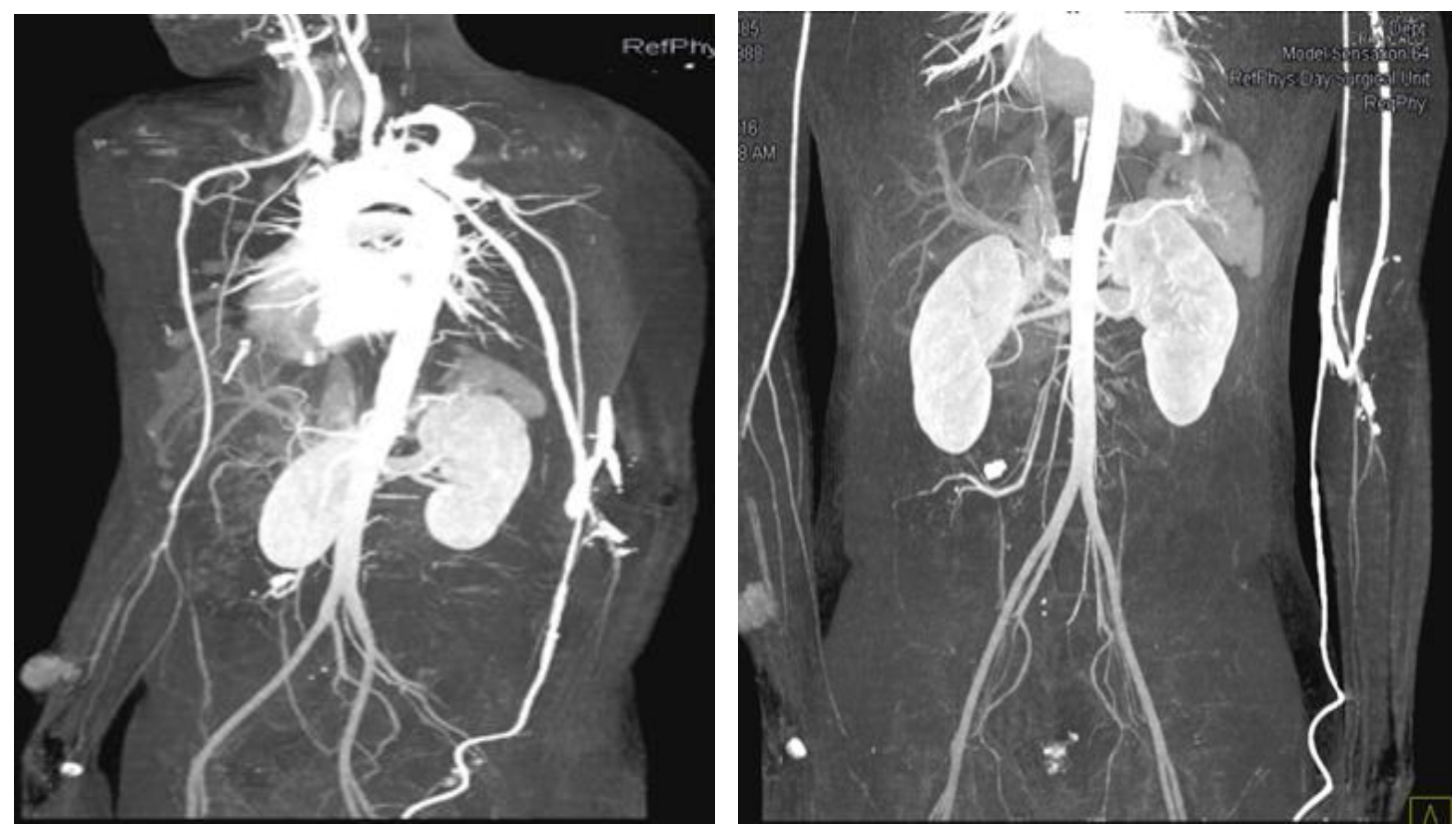

Figure 6 \&7. Ct angiogram shows Subcutaneous soft tissue mass seen at the dorsal aspect of forearm shows heterogeneous enhancement on the arterial phase with increased enhancement in the venous phase Hospital course and follow up:

Patient was referred to oncology after doing a CT chest and abdomen and confirming this was an isolated lesion, a decision was made for biopsy and surgical excision. 


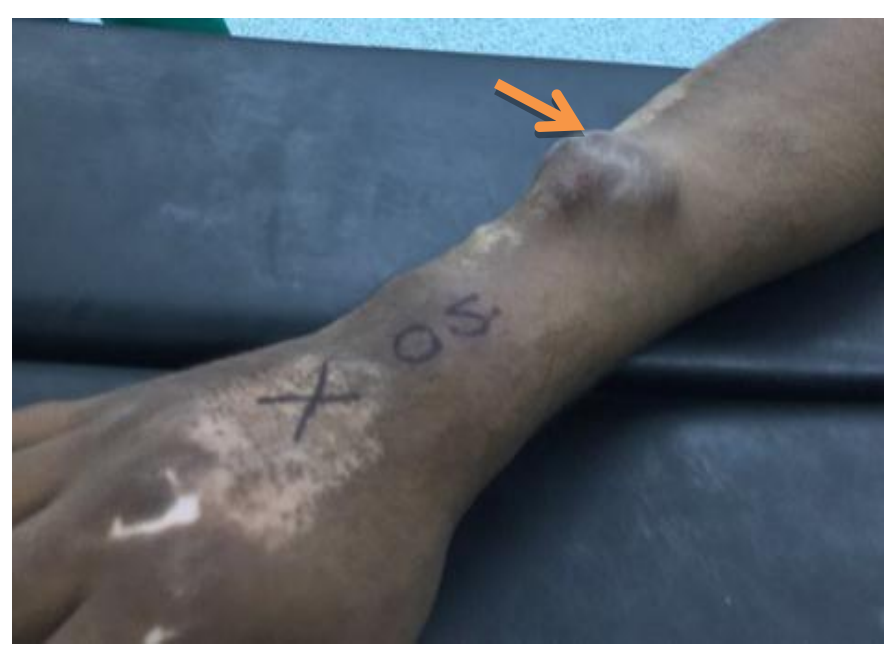

Figure 8. Shows Mass in Dorsal Forearm

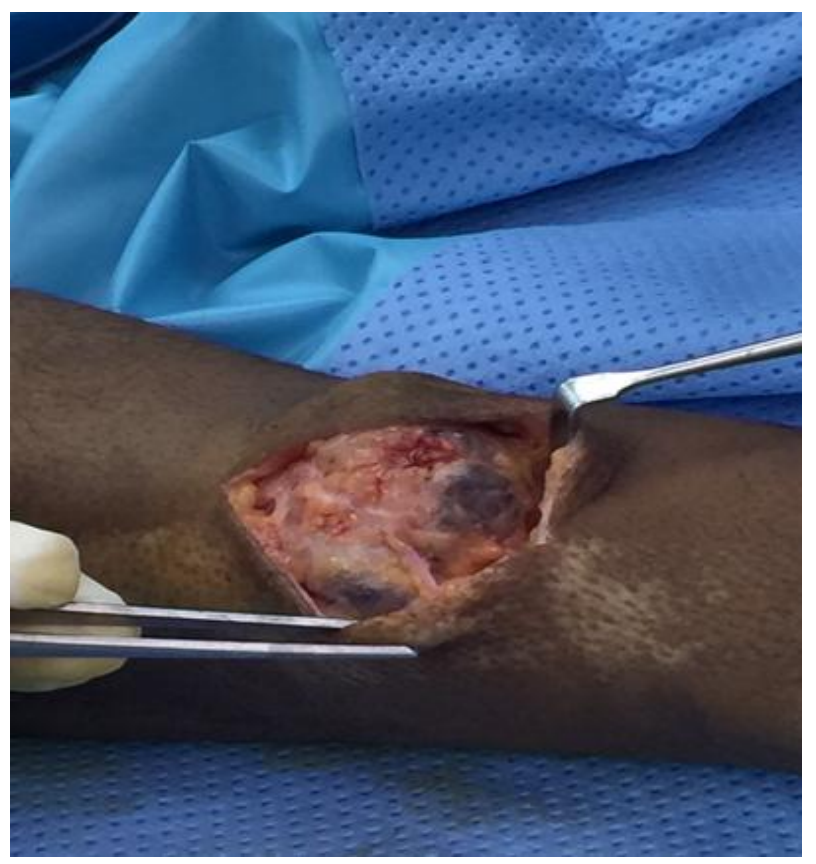




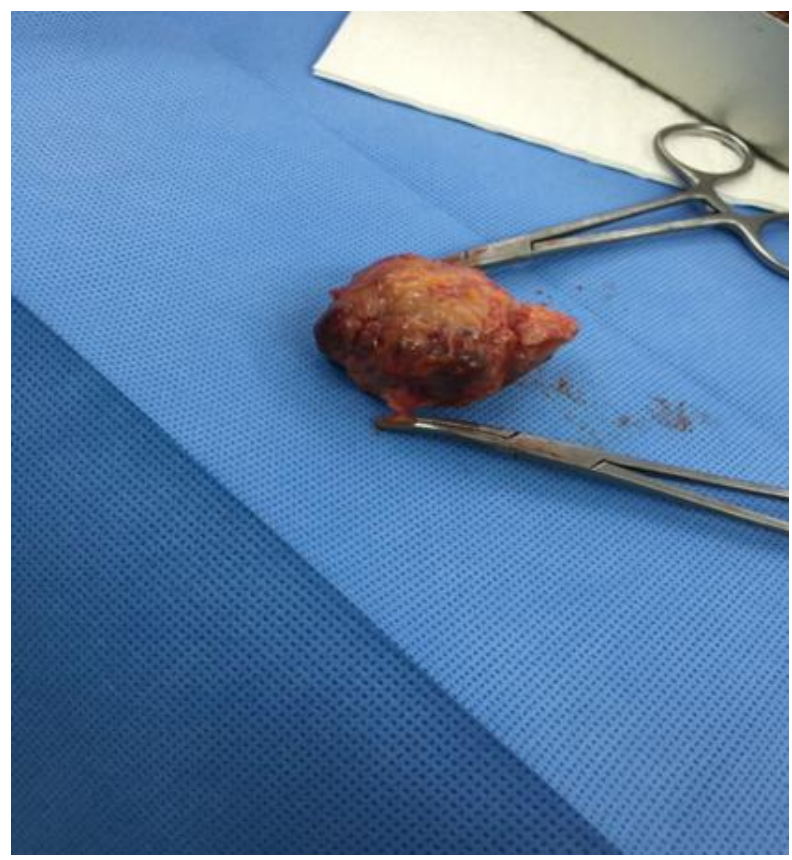

Figure 9. Intra-Operative; Shows Soft Tissue Tumor with Vascular Involvement Biopsy Taken.

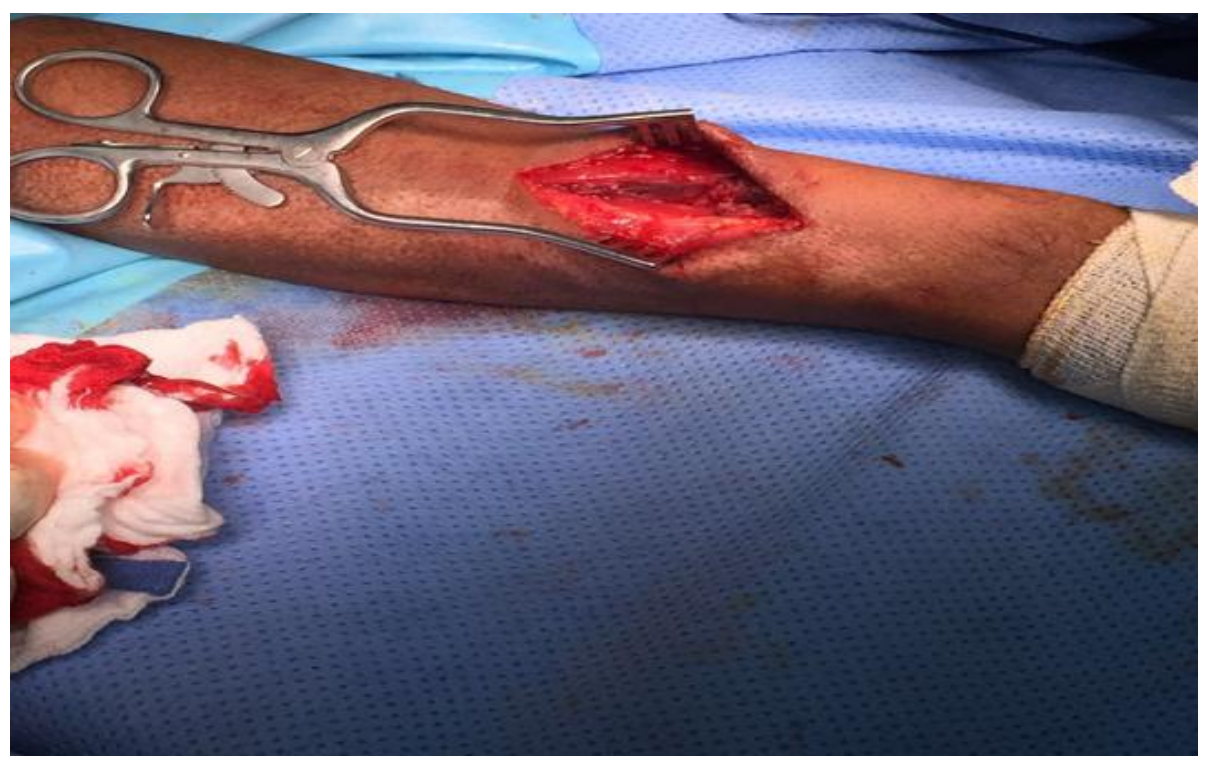

Figure 10. Intra-Operative; Shows Complete Excision of the Tumor.

Post-operative Histopathology and Follow up

overall histological features of this case suggests angiomatoid fibrous histiocytoma. It was solid with lobulated sheets of plump to spindled cells having histiocytic features adjacent to areas of hemorrhage. Patient was seen in our OPD post-operative 2 weeks later, suture was removed, no complain serial fellow up in OPD up to one year with no recurrence of such tumor.

\section{Morphology of AFH}

Grossly AFH is firm and circumscribed, and cross sectioning may reveal numerous blood-filled cystic spaces. Microscopically, the lesion is well demarcated and multi- nodular, often with a thick fibrous pseudocapsule (Figure 11) and comprising sheets, short fascicles, and occasionally storiform distributions of ovoid to spindle cells with bland, vesicular nuclei (Figures 12 and 13). Mitoses are in- frequent. Multifocal hemorrhage is seen in the majority of cases, leading to the formation of large non-endothelial- lined cystic cavities containing blood (Figure 14). Up to $80 \%$ of cases show a surrounding dense lymphoplasma cytic infiltrate ${ }^{5}$ (Figure 15), in some cases with germinal center formation, which may mimic the features of meta- static tumor within lymph node. However, variants to the features described are frequent, with only the multinodular pattern of ovoid to spindle 
cells being a consistent finding; the other characteristics can be absent or unrepresented in histologic sections. Giant cells are occasionally seen (Figure 16), and nuclear pleomorphism and increased mitotic activity may be noted.
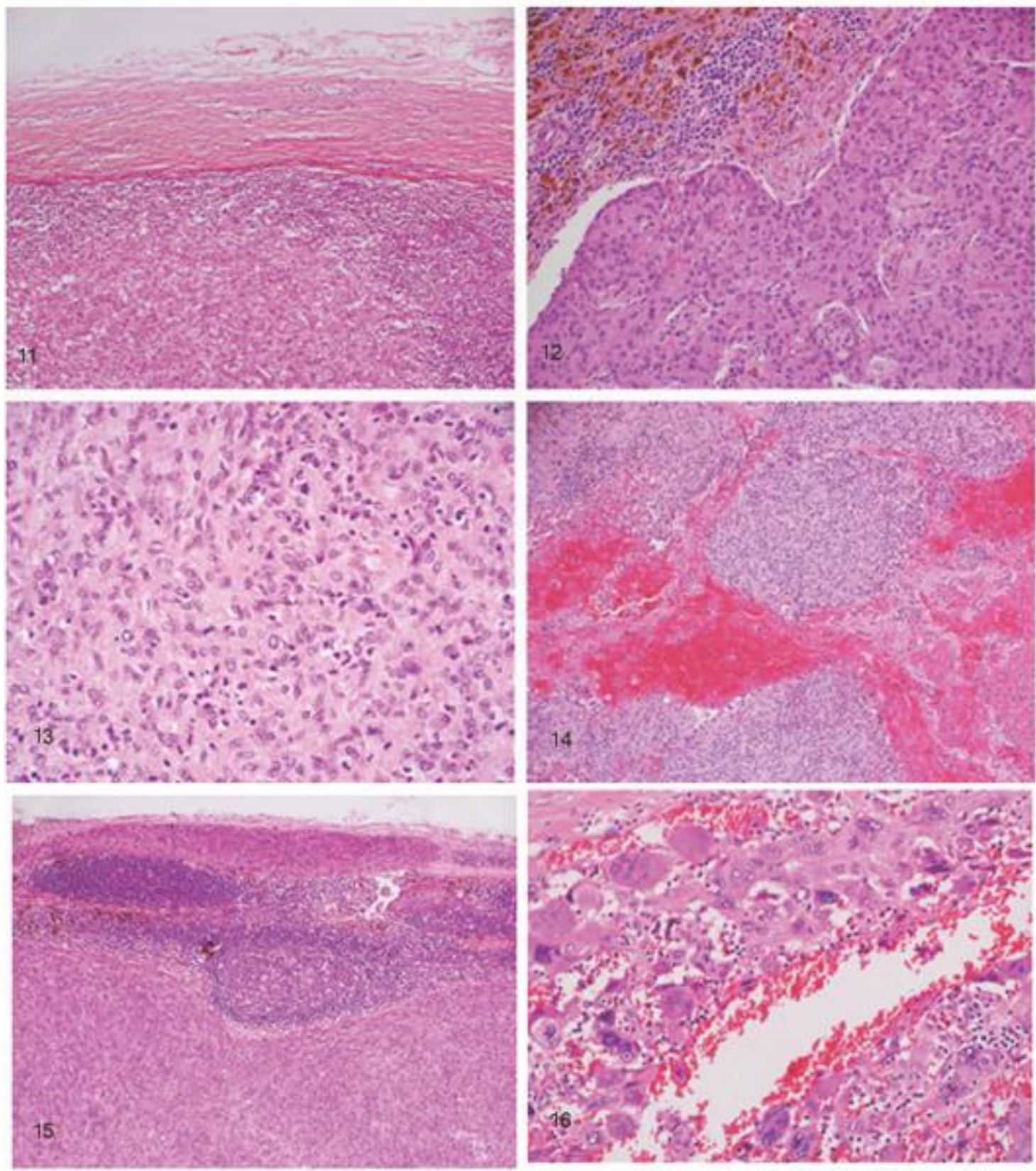

Figure 11. Angiomatoid fibrous histiocytoma: a thick fibrous pseudocapsule is present (hematoxylin-eosin, original magnification 200).

Figure 12. Interface between lymphoplasmacytic infiltrate and tumor (hematoxylin-eosin, original magnification 200).

Figure 13. Lesional cells show bland, vesicular nuclei (hematoxylin-eosin, original magnification 400).

Figure 14. Multiple nodules of spindle and ovoid cells with cavernous blood-filled spaces (hematoxylin-eosin, original magnification 100).

Figure 15. The dense lymphoplasmacytic infiltrate may mimic the appearance of a lymph node (hematoxylin-eosin, original magnification 40).

Figure 16. Occasional cases may show giant cells (hematoxylin-eosin, original magnification 200). 


\section{Discussion}

Angiomatoid fibrous histiocytoma (AFH) is a rare soft tissue neoplasm first described by Enzinger in 1979. WHO in 2002 removed AFH as a subtype of malignant sarcoma and placed it in the category of tumors of uncertain differentiation. Majority of the cases occur in the extremities although lesions occurring in the head and neck region (10\%) and trunk have been reported (Santa et al., 1981; Regezi et al., 1987). This tumor typically affects children and young adults, and it presents as a painless, slowly growing subcutaneous soft tissue mass that is usually located in the extremities and less commonly in the trunk, head and neck (Fanburg-Smith \& Miettinen, 1999). Clinically, it may resemble hematoma, hemangioma or a benign cyst, as it was in our case (Billings \& Folpe, 2004).

Death due to the disease is extremely rare in patients with angiomatoid fibrous histiocytoma (Grossman et al., 1996). Local recurrences have been reported in $12 \%$ of cases in one large study, and it is common in cases with infiltrating margins, involvement of the skeletal muscle and the head and neck region. ${ }^{2} \mathrm{~A}$ wide surgical excision and a careful follow-up are recommended since there is no evidence that adjuvant therapy may play a role in the primary management of this tumor.

\section{Differential Diagnosis}

The differential diagnosis encompasses a heterogeneous spectrum of conditions from reactive lesions to both benign and malignant neoplasms. It is important to establish a correct diagnosis to avoid overtreatment when misinterpreted as a more aggressive tumor and also, despite its relevant indolence, to avoid underdiagnosis because of its potential for recurrence and metastasis. Nodular Kaposi sarcoma is a possible mimic, with its circumscription, dermal location, bland spindle cell morphology, and in particular prominent vascular spaces. The pseudoangiomatoid spaces in AFH, however, lack an endothelial lining, instead being lined by flattened tumoral cells. This feature and the absence of CD34 and HHV8 should make distinction apparent. Aneurysmal benign fibrous histiocytoma is another superficial lesion occurring in young adults. This is centered predominantly within the dermis and generally has a more polymorphous cellular pattern including giant cells and siderophages. Moreover, it should still retain typical features of dermatofibroma, such as epidermal hyperplasia and peripheral collagen bundles, and is desmin negative.

Metastatic tumor to lymph node (including metastasizing cellular fibrous histiocytoma) may enter the differential diagnosis at first glance, although closer inspection of AFH reveals that germinal centers are randomly arranged around the tumor, and true nodal architecture such as subcapsular and medullary sinuses are absent. Other tumors particular to the lymph node, such as palisaded myofibroblastoma and inflammatory pseudotumor, may be considered. Of these, the former shows delicate palisading spindle cells and thick bands or bundles of collagen fibers ("amianthoid fibers") and is desmin negative; the latter, although occasionally seen to express desmin, lacks the pseudovascular spaces of AFH. Spindle cell hemangioma typically occurs in the dermis and subcutis of the distal extremities. It contains cavernous vascular spaces, but in contrast to AFH these are lined by an attenuated layer of endothelial cells. It is also poorly circumscribed and often contains epithelioid cells, many of which contain intracytoplasmic vacuoles. The bland ovoid to spindle cells of AFH can sometimes also be mistaken for granulomas, although the more solid nature of the tumor and blood-filled spaces should make distinction possible.

Desmin positivity can be seen in occasional dendritic cells in both localized and diffuse tenosynovial giant cell tumors (Folpe et al., 1998). Although AFH may contain giant cells, this should not be a source of confusion, as the morphology of giant cell tumors, with their heterogeneous cellular composition including foamy macrophages, is characteristic.

\section{Conclusion}

Angiomatoid fibrous histiocytoma is a neoplasm of intermediate biologic potential. Most often, the extremities are the most common site of involvement, followed by the trunk and head and neck of children and young adults. Its rarity may lead to misdiagnosis as either a reactive lesion or a benign or higher-grade tumor. This tumor has a relatively rare metastasis and its overall clinical outcome is excellent. It is clinically important to accurately diagnose this tumor so that wide local excision may be performed and patients may be appropriately monitored and followed up to ensure that no signs or clinical evidence of tumor recurrence occurs.

\section{References}

Enzinger, F. M. (1979). Angiomatoid malignant fibrous histiocytoma: a distinct fibrohistiocytic tumor of children and young adults simulating a vascular neoplasm. Cancer, 44, 2147-2157. https://doi.org/10.1002/1097-0142(197912)44:6<2147::AID-CNCR2820440627>3.0.CO;2-8 
Costa, M. J., \& Weiss, S. W. (1990). Angiomatoid malignant fibrous histiocytoma. A follow-up study of 108 cases with evaluation of possible histologic predictors of outcome. Am J Surg Pathol, 14, 1126-1132. https://doi.org/10.1097/00000478-199012000-00004

Fanburg-Smith, J. C. (2002). Angiomatoid fibrous histiocytoma. In: Fletcher CDM, Unni KK, Mertens F (eds). World Health Organization Classification of Tumours. Pathology and Genetics. Tumours of Soft Tissue and Bone. Lyon: IARC, 194-195

Athanasou, N. A. (1999). Colour Atlas of Bone, Joint, and Soft Tissue Pathology. Oxford University Press: New York.

Fletcher, C. D. (2006). The evolving classification of soft tissue tumours: An update based on the new WHO classification. Histopathology, 48, 3-12. https://doi.org/10.1111/j.1365-2559.2005.02284.x

Bauer, A., Jackson, B., Marner, E., \& Gilbertson-Dahdal, D. (2012). Angiomatoid fibrous histiocytoma: A case report and review of the literature. J Radiol Case Rep, 6, 8-15. https://doi.org/10.3941/jrcr.v6i11.932

Santa, Cruz, D. J., \& Kyriakos, M. Aneurysmal. (1981). Fibrous histiocytoma of the skin. Cancer, 47, 2053-61. https://doi.org/10.1002/1097-0142(19810415)47:8<2053::AID-CNCR2820470825>3.0.CO;2-A

Regezi, J. A., Zarbo, R. J., Tomich, C. E., Lloyd, R. V., Courtney, R. M., \& Crissman, J. D. (1987). Immunoprofile of benign and malignant fibrohistiocytic tumors. $J$ Oral Pathol, 16, 260-5. https://doi.org/10.1111/j.1600-0714.1987.tb01490.x

Fanburg-Smith, J. C., Miettinen, M. Angiomatoid. (1999). "malignant" fibrous histiocytoma: a clinicopathologic study of 158 cases and further exploration of the myoid phenotype. Hum Pathol, 30, 1336-43. https://doi.org/10.1016/S0046-8177(99)90065-5

Billings, S. D., \& Folpe, A. L. (2004). Cutaneous and subcutaneous fibrohistiocytic tumors of intermediate $\begin{array}{lllll}\text { malignancy: an } \quad \mathrm{Am} \text { unte. } J \text { Dermatopathol, } & \text { 26, }\end{array}$ https://doi.org/10.1097/00000372-200404000-00035

Grossman, L. D., White, R. R. 4th, \& Arber, D. A. (1996). Angiomatoid fibrous histiocytoma. Ann Plast Surg, 36, 649-51. https://doi.org/10.1097/00000637-199606000-00016

Folpe, A. L., Weiss, S. W., Fletcher, C. D., \& Gown, A. M. (1998). Tenosynovial giant cell tumors: evidence for a desmin-positive dendritic cell subpopulation. Modern pathology: an official journal of the United States and Canadian Academy of Pathology, Inc. 10, 939-44.

\section{Copyrights}

Copyright for this article is retained by the author(s), with first publication rights granted to the journal.

This is an open-access article distributed under the terms and conditions of the Creative Commons Attribution license (http://creativecommons.org/licenses/by/4.0/). 